Unternehmenskommunikation im neuen Jahrtausend

\section{Die Zukunft erfordert eine nachhaltige Kommunikation}

\author{
Kommunikation ist in den meisten Unternehmen bisher nur \\ eine Disziplin von vielen. Doch die Zeiten ändern sich: \\ Langfristiger Unternehmenserfolg hängt zunehmend von der \\ Qualität des Austauschs mit den Stakeholdern ab. Ein Plädoyer \\ dafür, warum Kommunikation in Zukunft ein zentrales Element \\ der Unternehmenssteuerung sein sollte. Von Marcel Bernard
}

D ie Kommunikations- und Informationstechnologien haben sich in den letzten zehn Jahren radikal verändert. Deshalb kommunizieren die Menschen heute anders. Das betrifft Kunden und Mitarbeiter ebenso wie Investoren und die Öffentlichkeit. Ein Beleg für diese Behauptung sind die Marktwerte von Unternehmen wie Google, Facebook, Amazon oder Apple. Für sie ist Kommunikation Produkt, Alleinstellungsmerkmal und Vertriebskanal zugleich.

Viele klassische Unternehmen sind sich durchaus bewusst, dass diese Entwicklungen auch ihr Geschäft beeinflussen werden. In den Geschäftsleitungen fehlt aber oft die nötige Priorität, sich das komplexe Wissen über Zusammenhänge, Hintergründe und Wirkungen anzueignen. Dieses Wissen ist aber notwendig, um Chancen und Risiken fundiert bewerten zu können. So herrscht oft Ratlosigkeit gepaart mit blindem Aktionismus vor.

\section{Welche Rolle spielte Kommunikation bisher?}

In den meisten Unternehmen war Kommunikation vor allem ein Mittel zum Zweck. Und damit ein wichtiger Erfüllungsgehilfe bei der Profitmaximierung. Das betraf alle kommunikativ ausgerichteten Bereiche wie Vertrieb, Marketing, Öffentlichkeitsarbeit und die interne Kommunikation.

Profitmaximierung folgt bekanntlich einer klaren Maxime: so günstig wie mög- lich einkaufen, so teuer wie möglich verkaufen. Dies geht oft zulasten von Mensch und Umwelt. Als das Internet noch nicht zu unserem Alltag gehörte, erfuhr die Öffentlichkeit nur selten von solchen Vergehen. Marketing, Vertrieb und Öffentlichkeitsarbeit konnten so meist ungestraft falsche Versprechen geben.

Erst wenn ein Skandal ans Licht kam, wurde Kommunikation zur Chefsache. Den Unternehmensführern fiel es schwer, sich erfolgreich mit einer kritischen Öffentlichkeit auseinanderzusetzen. Kein Wunder, war doch Kommunikation lediglich eine Abteilungsaufgabe, die jedem Stakeholder immer die gerade passende Botschaft zu vermitteln hatte. Transparenz, Wertschätzung und Dialogfähigkeit waren eben keine Elemente der Kommunikationskultur. Der Erfolg gab den Unternehmen dennoch lange Zeit Recht. Deshalb ließen sich auch die vielen Kommunikationsabteilungen mit ihren teueren Stäben und Maßnahmen rechtfertigen. Von der schwierigen und oft kontraproduktiven internen Abstimmung zwischen den Abteilungen ganz zu schweigen.

Die Rolle der Kommunikation war also bisher klar definiert. Sie sollte helfen, den Gewinn zu maximieren.

\section{Der Markt ändert sich}

Zwei sich wechselseitig beeinflussende Faktoren haben die Rahmenbedingungen für Unternehmen in den letzten Jahren drastisch verändert. Zum einen das stetig wachsende gesellschaftliche Bewusstsein für ökologische und soziale Verantwortung. Zum anderen die unwiderrufliche Vernetzung der Welt mit in Echtzeit verfügbaren Informationen. Konsumenten nutzen die Kommunikationstechnologien aber nicht nur privat, etwa für Kaufentscheidungen, sondern mischen sich auch aktiv ins öffentliche Geschehen ein. Heute kann jeder Mensch mit geringem Aufwand sein eigener Produzent, Verlag oder TV-Sender werden. Die klassischen Medien haben damit ihr Monopol auf Meinungsbildung verloren.

Hintergründe über unmenschliche Produktionsbedingungen oder ein rücksichtsloses Management erreichen heute schneller eine große Öffentlichkeit. Diese Dynamik hat großen Einfluss auf das Verhalten von Unternehmen, die bisher eine mehr oder weniger totale Kontrolle ihrer Botschaften gewohnt waren.

\section{Konsequenzen für Unternehmen}

Die neuen Rahmenbedingungen eröffnen Chancen, bergen aber auch Risiken. Wer nichts zu verbergen hat, kann die Kraft seiner Kontakte nutzen, um die klassische Bewerbung der Produkte durch den besten und günstigsten Vertriebsweg zu ersetzen, den Empfehlungen. Die Nutzung dieser vernetzten Kommunikation für die Entwicklung von Geschäfts- und Produktstrategien steht erst am Anfang. Gleichzeitig ist die Verlockung groß, die neue Kommunikation wie die alte $\mathrm{zu}$ nutzen und sein Unternehmen lediglich grün anzumalen. Dieses sogenannte Greenwashing kann durchaus eine Zeit lang gutgehen. Halten aber Unternehmensführung und Produkte die Werbeversprechen nicht ein, kann sich diese Nachricht wie ein Lauffeuer verbreiten und damit das wichtigste Gut einer Marke, das Vertrauen, zerstören.

Die meisten Unternehmer werden nur dann ökologische und soziale Verantwortung übernehmen, wenn sie sich langfristig ökonomisch rechnet.

Um eine strategische Entscheidung zu fällen, ist eine fundierte Grundlage nötig. Sie sollte jedoch nicht alleine durch das 
Management oder Berater geschaffen werden, sondern durch die Stakeholder, die die Entwicklung des Unternehmens maßgeblich beeinflussen. Die Anforderungen und Leistungsbereitschaft der Stakeholder zu kennen und ihre positiven wie auch negativen Potentiale auf die Unternehmensentwicklung zu bewerten, ist der erste wichtige Schritt.

Damit sich die Stakeholder trotz oft sehr unterschiedlicher Interessen mit dem Unternehmen identifizieren und seine Entwicklung fördern, müssen ein paar Grundregeln eingehalten werden.

I Ehrlichkeit: Solange die Motivation moralisch sauber ist, dürfen auch unbequeme Dinge gesagt und Fehler gemacht werden.

- Verbindlichkeit: Große Versprechungen sind passé. Lieber weniger versprechen, dies aber halten.

I Dialog: Wissen, Informationen und Meinungen sammeln und tauschen. Das betrifft Vertrieb, Produktentwicklung und Unternehmensorganisation.

I Transparenz: Einblick in den Businessplan und Patente wünschen sich die Kunden und Mitarbeiter gar nicht, sehr wohl aber in Strategie und Produktionsprozesse.

\section{Was kann ein Unternehmen konkret unternehmen?}

Hält das Unternehmen die oben genannten Grundsätze nicht ein, droht der gegenteilige Effekt. Das Unternehmen verliert seine Glaubwürdigkeit und gefährdet seine Existenzgrundlage.

Um wirklich etwas verändern zu können, muss Kommunikation auf Vorstands- oder Geschäftsführerebene verankert werden. Dies kann in Form einer Stabstelle geschehen. Sie gestaltet und steuert abteilungs- und hierarchieübergreifend die Kommunikation mit den Stakeholdern. Dabei ist die Stabstelle nicht von den vorhandenen Kommunikationsabteilungen abhängig.

Die Stabstelle sollte folgende Aufgaben haben:

I Wissen über gesellschaftliche, prozessuale und technologische Entwicklungen vermitteln.

- Ein breites Verständnis über die Wirkung von Kommunikation schaffen.

- Die Kommunikationskultur inklusive einer messbaren Kommunikationsstrategie entwickeln.

I Die Corporate Identity des Unternehmens kreieren und umsetzen.

I Abteilungsübergreifende Kommunikationsprozesse erarbeiten und koordinieren. Beispiel: Welche Abteilungen durchläuft ein Kunde in dem Unternehmen, vom Vertrieb über den Service bis zum After Sale.

- Die Fachabteilungen als interner
Dienstleister dabei unterstützen, eine nachhaltige Kommunikation mit den jeweiligen Zielgruppen zu entwickeln.

- Zwischen den verschiedenen Stakeholderinteressen vermitteln und die Stakeholderdialoge moderieren.

Die Mitarbeiter einer solchen Stabstelle benötigen neben der fachlichen und methodischen Kompetenz etwas, das man „gesunder Menschenverstand“ nennen kann, kombiniert mit Konsequenz und Disziplin.

\section{Für eine glaubwürdige Kommunikation}

Der Weg zu einer dialogorientierten und glaubwürdigen Kommunikation kann durchaus steinig sein. Eine Verpflichtung zu Ehrlichkeit und Transparenz lässt wenig Raum für Eitelkeiten und persönliche Interessen. Entscheidungsprozesse dauern länger und müssen Konflikte aushalten. Und es ist nicht immer leicht, das richtige Maß zwischen $\mathrm{zu}$ viel und zu wenig Beteiligung zu finden. Diese Investition zahlt sich aber aus. Denn Produkte und Dienstleistungen erfüllen besser die Bedürfnisse der Kunden. Zusammen mit einer hohen gesellschaftlichen Akzeptanz resultiert dies in ökonomischem Erfolg. Die Mühe lohnt sich also.

I AUTOR + KONTAKT

Abbildung 1: Stakeholder Kommunikation im Unternehmensumfeld

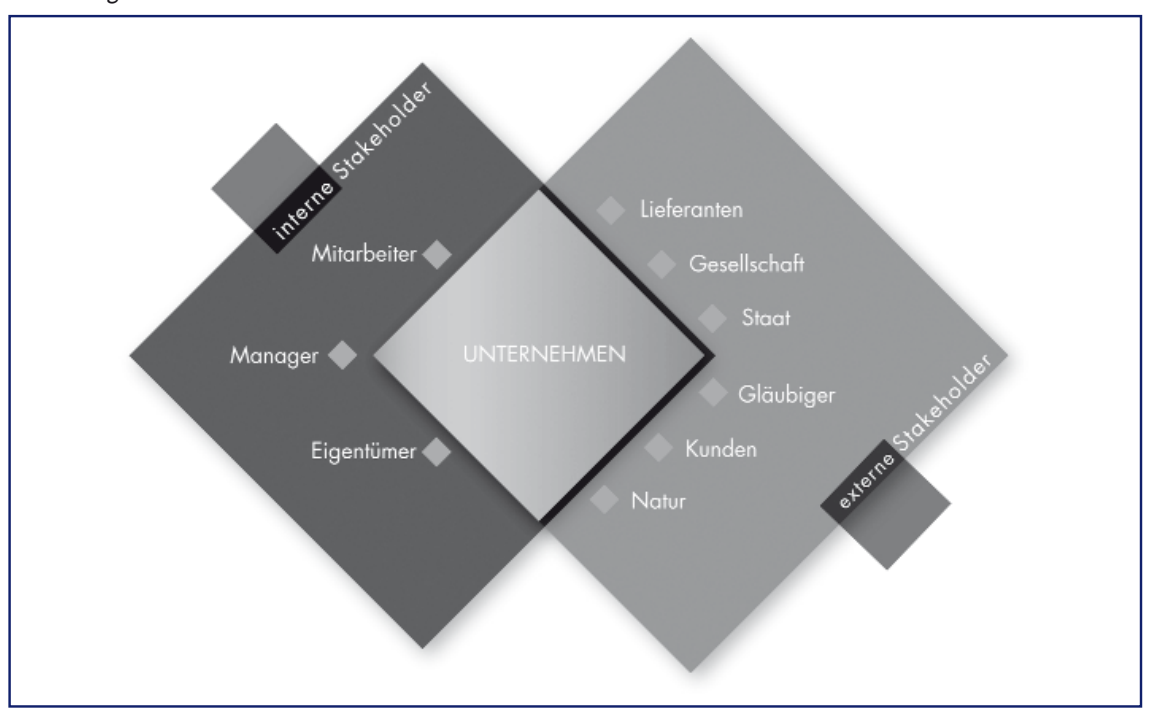

Quelle: Eigene Abbildung 


\section{Lizenzhinweis}

Die Beiträge in ÖkologischesWirtschaften werden unter der Creative-Commons-Lizenz "CC 4.0 Attribution Non-Commercial No Derivatives" veröffentlicht. Im Rahmen dieser Lizenz muss der Autor/Urheber stets genannt werden, das Werk darf nicht bearbeitet, abgewandelt oder in anderer Weise verändert und außerdem nicht kommerziell genutzt werden. Die digitale Version des Artikels bleibt für zwei Jahre Abonnent/innen vorbehalten und ist danach im Open Access verfügbar. 